Military Technical College Kobry El-Kobbah, Cairo, Egypt

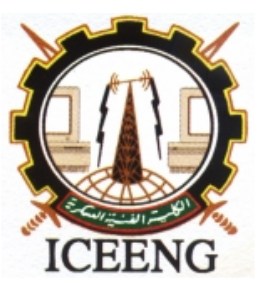

\author{
$8^{\text {th }}$ International Conference \\ on Electrical Engineering \\ ICEENG 2012
}

\title{
Effect of Mutual Coupling on MIMO capacity of a Microstrip Antenna Array
}

By
M. M. Aldemerdash*
A. M. Abdin**
A. A. Mitkees***
H. A. Elmikati****

\section{$\underline{\text { Abstract: }}$}

The effect of mutual coupling on the capacity of Multiple-Input-Multiple Output (MIMO) antenna systems is demonstrated by considering a linear array of a four element line fed microstrip patch array. This array is designed at ISM band with frequency of $2.45 \mathrm{GHz}$ on FR4 Epoxy substrate with relative permittivity $\left(\varepsilon_{\mathrm{r}}\right)$ of 4.4. The simulation results obtained with this array are compared with the results of a similar array having 2 columns and 4 rows spiral Electromagnetic Band Gap (EBG) structure. The spacing between array elements is variable at the transmitting end and fixed at the receiving end with $0.5 \lambda$. Array impedance matrices are obtained from simulation results generated by the Ansoft designer software and then used in the calculation of the required coupling matrix. The one-ring channel model is used to compute the correlation between paths and mean capacity of the MIMO system for equal power (EP) and waterfilling (WF) schemes at SNR of $20 \mathrm{~dB}$. A maximum mutual coupling reduction of $17 \mathrm{~dB}$ is achieved as a result of using spiral EBG structure. For element spacing in the range $(0.48 \lambda-0.6 \lambda)$, which is suitable for the design of antenna arrays, the mutual coupling reduces the correlation level from the ideal case by about 0.15 and the mean capacity by $(0.1-0.42) \mathrm{b} / \mathrm{s} / \mathrm{Hz}$. The reduction of the mutual coupling by spiral EBG structure improves the correlation and the mean capacity that their values are reaching the ideal.

* College of Engineering, Mansoura University, Mansoura, Egypt

** Yanbu Industrial College, Yanbu, Saudi Arabia

*** MTC, Cairo, Egypt

**** College of Engineering, Mansoura University, Mansoura, Egypt 


\section{Keywords:}

MIMO systems, antenna arrays, mutual coupling, EBG structure, channel capacity, onering model, SCM model, Kroncker model

\section{Introduction:}

In modern wireless communication systems, high data rate is required over band-limited channels. MIMO systems that utilize multiple antennas to increase channel capacity without scarifying additional spectrum or transmitted power have received a growing amount of interest in recent years. Theoretically and under ideal conditions the capacity of the MIMO system increases with the number of antennas used. However this only stands when the signals at the transmitting and receiving antennas are totally uncorrelated. When correlation exists the number of antenna elements that can lead to an increase in the capacity is limited. Adding antenna elements beyond this limit can even cause the capacity of the system to degrade [1].

The capacity of the MIMO system depends mainly on the channel and the antenna characteristics. The capacity can be improved by proper design of antenna elements and choosing appropriate array configuration. Therefore, it is important to know how various array configurations are performing in the case of MIMO systems. It is shown that the directive elements give more capacity than omni-directional elements. There is a considerable change in capacity due to the effect of mutual coupling. When the spacing between the elements is less than $0.5 \lambda$, the effect of mutual coupling is increased [2].

The MIMO channel modeling, has two main approaches, physically based and nonphysically based modeling. The physical models describe the MIMO channel (or its distribution) via some physical parameters such as Doppler frequency, angle of departure (AOD), angle of arrival (AOA) and angle spread. The non-physical models primarily rely on the statistical characteristics of the MIMO channels obtained from the measured data. The one-ring model and Spatial Channel Model (SCM) are examples of the physical channel models, while the Kronecker channel model is an example of the non physical models. As compared to independent identically distributed (i.i.d.) channel model, the one ring model, SCM model and Kronecker model takes into account the inter-element distance in transmitter and receiver arrays which makes mutual coupling investigation feasible [1-3]. The one ring-model is used in this work for its simplicity and sufficient to demonstrate the effectiveness of the mutual coupling on the MIMO capacity.

Previous works have shown the effect of mutual coupling on system capacity of a MIMO 
system for various antenna elements. In [1], the dependence of the capacity of $2 \mathrm{xM}$ MIMO systems on the mutual coupling is investigated using Kronecker channel model. Linear arrays of microstrip rectangular antennas are considered. Here, the mutual coupling was calculated from formulas obtained experimentally. In [2] and [3], the effect of mutual coupling on the capacity of a MIMO system was evaluated for spatial channel model and one ring model scenarios. The antennas are made of thin wire dipoles and the mutual coupling was calculated using the induced EMF method. In [4], a two elements microstrip antenna array was used at the transmitter and receiver ends, a mushroom EBG structure was also used to reduce the mutual coupling between array elements and the simulation was performed on CST Microwave Studio. Capacity results are obtained via Monte Carlo simulation.

The aim of this paper is to study the effect of the mutual coupling on the capacity of MIMO system. The arrays used at the transmitting and receiving ends are four element microstrip antenna array arranged in side-by-side configuration. The spiral EBG structure is used to reduce the mutual coupling effect. The Capacity results are obtained via the one-ring model.

The paper is organized as follows. The system model is presented in section 2; the microstrip antenna array is introduced in section 3, whereas the mutual coupling and channel capacity formulas are reviewed in section 4 and 5, respectively. Results and discussions are presented in section 6. Finally, conclusions are reported in section 7.

\section{System Model:}

Consider a narrow-band single user MIMO system with $\mathrm{N}_{\mathrm{T}}$ transmitting and $\mathrm{N}_{\mathrm{R}}$ receiving antennas. The overall MIMO input-output relationship can be represented in vector notation as in [3] and [5]

$y=H x+n$

where $y$ is the $N_{R} \times 1$ received signal vector, $x$ is the $N_{T} \times 1$ transmitted signal vector, $n$ is the $\mathrm{NR} \times 1$ zero-mean complex Gaussian noise vector with independent, equal variance real and imaginary parts, and $\mathrm{H}$ is the $\mathrm{N}_{R} \times \mathrm{N}_{T}$ normalized channel matrix. Each element $h_{i, j}$ represents the complex gains between the $j^{\text {th }}$ transmit and $i^{\text {th }}$ receive antennas. In principle, any channel model that accurately includes the spatial dimensions could be used to investigate the correlation properties of two spatially separated antennas. 
The one-ring channel model is the proposed model for this work because of its simplicity and at the same time includes the spatially dimensions [2], [5]. It is a widely used model for outdoor environment. In this model, the base station (BS) is assumed to be elevated, which means that there is no obstruction by local scatterers while the mobile station (MS) is surrounded by scatterers lying in a circular disc with uniform distribution. The scatterers are assumed to be omni-directional re-directed objects to reflect the plane wave directly to the receiving antenna without influencing other scatterers. Only those rays that reflect once by the scatterer are considered. Figure 1illustrates the scatter disc and the orientation of the mobile and base station.

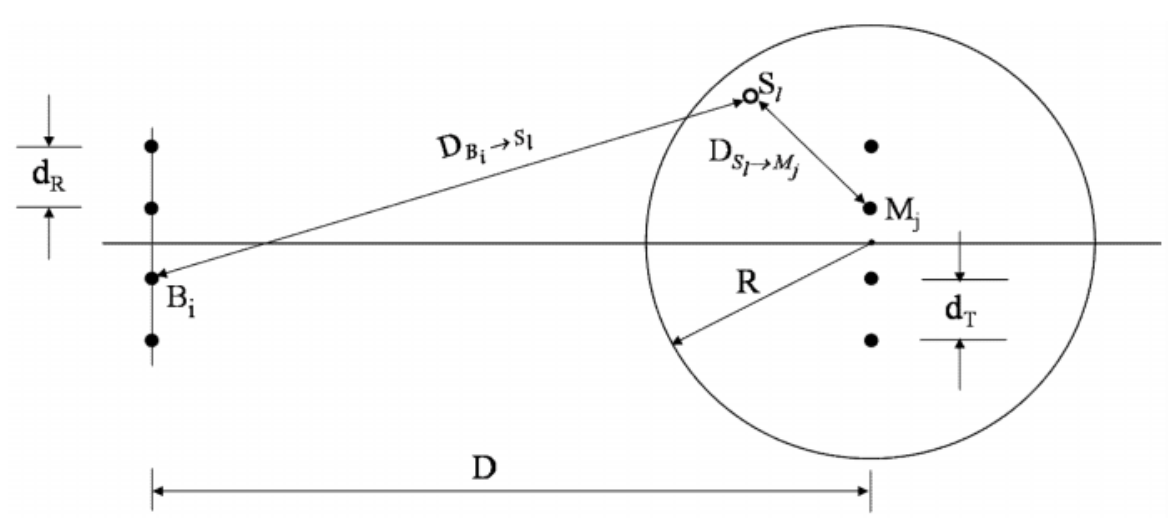

Figure (1): Geometry of the one-ring channel model

Based on this model, the elements of the channel matrix $h_{i, j}$ are generated as. Assume $L$ scatterers $\boldsymbol{S}_{l}, l=1,2, \ldots, \mathrm{L}$, are uniformly distributed on a disc of radius $\mathrm{R}$, which is around the mobile. Usually $\mathrm{R}$ is assumed to be smaller than the distance (D) between the transmitter (MS) and the receiver (BS). Furthermore, both R and D are assumed to be large compared with the antenna space $d_{T}$ and $d_{R}\left(\{D>R\} \gg \max \left(d_{T}, d_{R}\right)\right.$ ). Considering a single transmission path from $\mathrm{j}^{\text {th }}$ transmit antenna to $\mathrm{i}^{\text {th }}$ receive antenna intercepted and reflected by $l^{\text {th }}$ scatterer, the channel gain $\mathrm{h}_{\mathrm{ij}}$ is given by [5]

$h_{i j}=\sqrt{\frac{1}{L}} \sum_{l=1}^{L} \alpha_{l} e^{\left[-j \frac{2 \pi}{\lambda}\left(D_{B_{i} \rightarrow S_{l}}+D_{S_{l} \rightarrow M_{j}}\right)\right]}$

where $\mathrm{D}_{\mathrm{B}_{i} \rightarrow \mathrm{S}_{l}}$ and $\mathrm{D}_{\mathrm{S}_{l} \rightarrow \mathrm{M}_{j}}$ are the distances from base station antenna $i$ to scatterer $l$, and scatterer $l$ to mobile antenna $j$, respectively, as shown in Fig. 1. The scattering coefficient $\left(\alpha_{l}\right)$ from scatterer $l$ is modeled as a normal complex random variable with zero mean and unit variance. 


\section{The Microstrip Antenna Array:}

A line-fed microstrip patch was chosen as the radiating element of the proposed antenna array because of the inherent narrow bandwidth and the well known level of mutual coupling for a given separation. It gives a clear increase in bandwidth and reduction in mutual coupling [4]. It is designed to operate in the ISM band at frequency $2.45 \mathrm{GHz}$. The ground and the patch are made of copper. The dielectric material of the substrate is FR4 Epoxy with dielectric constant $\left(\varepsilon_{\mathrm{r}}\right)$ of 4.4, dielectric loss tangent $(\tan \delta)$ of 0.02 and thickness (h) of $1.588 \mathrm{~mm}$. The array consists of four identical elements arranged in side-by-side configuration at the $x$-axis and separated by a distance $\lambda / 2$ (approximately $61.25 \mathrm{~mm}$ ) where $\lambda$ is the design wavelength. This distance is from center to center of the antennas.

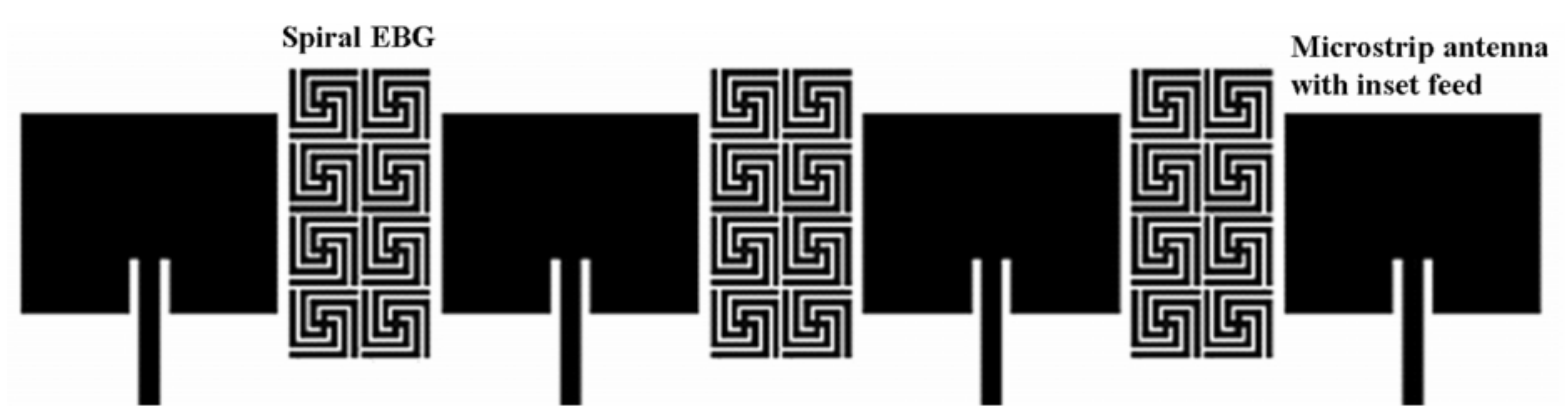

Figure (2): Four elements microstrip antenna array with spiral EBG substrate for MIMO channel transmitting and receiving ends

The used spiral EBG structure causes maximum reduction to the mutual coupling. It is periodic metal four-arm spiral branches on top of the substrate. I its center there is a via that shorts it to ground. The detailed design of the proposed structure is shown in Fig.2. It is already presented in [6] using the Ansoft Designer software. This design gives the mutual coupling between array elements and the optimum reduction of it when using two columns and four rows of spiral EBG structure. The parametric solution is started from $0.31 \lambda$ which is the minimum space between array elements without spiral EBG structure. On the other hand, the minimum distance when spiral EBG is used is $0.47 \lambda$.

The simulation was carried out for two cases. The first one is for fixed separation between array elements of $0.5 \lambda$ The second case is a parametric solution in the range of antenna separations started from the minimum distances to $1 \lambda$. Each of these two cases is simulated with and without spiral EBG structure. The impedance matrices for the two cases come from the simulation are used in the calculation of the coupling matrix discussed in section 4 . The mutual coupling and the mutual impedance between adjacent 
microstrip antennas are shown in Fig. 3. The maximum mutual coupling reduction is obtained at the operating frequency when the separation is $0.5 \lambda$.
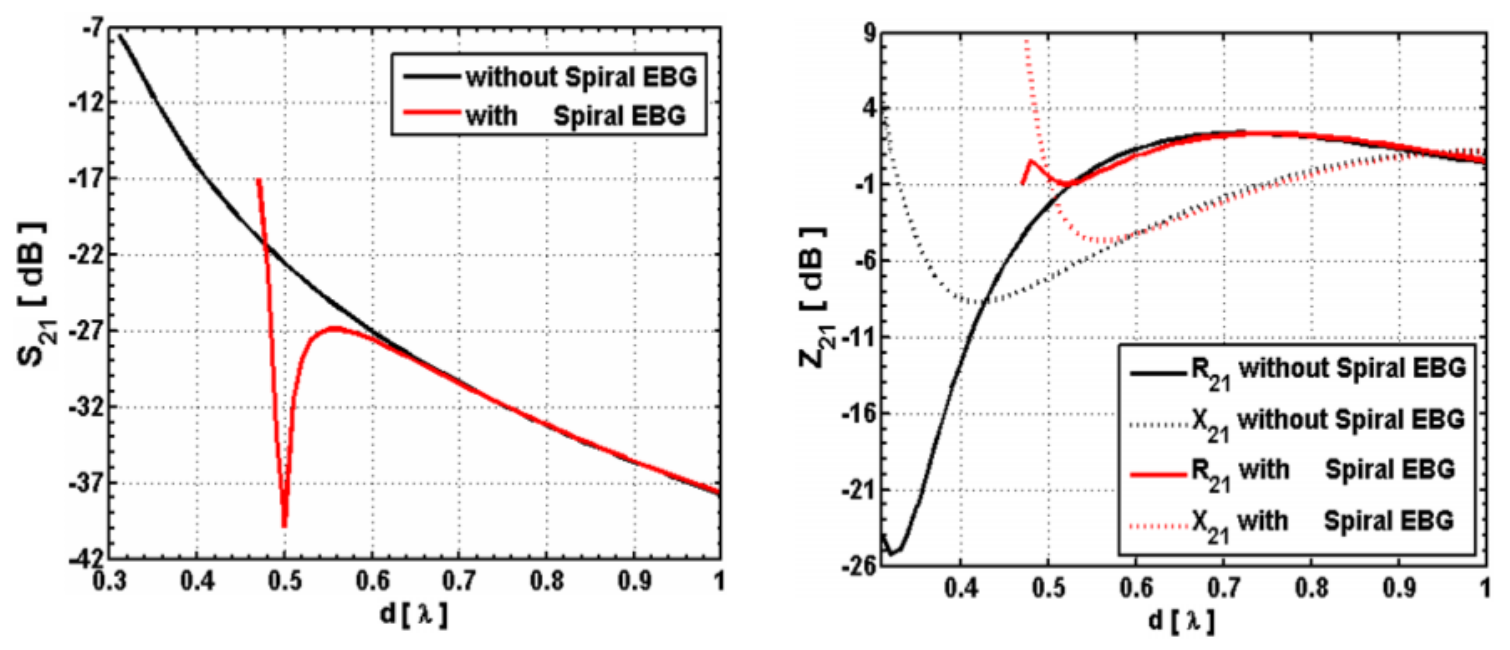

Figure (3): Mutual Coupling and mutual impedance of two elements microstrip antenna array

\section{Mutual Coupling:}

When antenna elements are placed close to each other, the electromagnetic field generated by the current flowing in one antenna causes a voltage induced in the neighboring antennas. This is called mutual coupling. For the microstrip patch antennas, the simulation by the Ansoft designer software is used to model the effect of mutual coupling in MIMO system. The mutual coupling due to the insertion of spiral EBG structure is taking into consideration in the transmission and reception sides.

The following coupling matrix $\mathrm{C}$ is modified from that given in $[3,5]$ to be suitable for the results obtained from simulation

$$
C=\left(\phi_{A}+\phi_{T}\right)\left(\not+\phi_{T}\right)^{-1}
$$

where $Z_{A}$ is the diagonal matrix of the mutual impedance matrix $\mathbf{Z}$ which represent the input impedance of the antennas in the array, $Z_{T}$ is the impedance of the measurement equipment at each element, chosen as the complex conjugate of $\mathbf{Z}_{\mathbf{A}}$ to obtain an impedance match for maximum power transfer.

The mutual impedance matrix $\mathrm{Z}$ is $N \times N$ matrix obtained from the Ansoft designer 
Software after optimum impedance matching at the operating frequency. It is given by:

$$
\phi=\left(\begin{array}{ccccc}
Z_{11} & Z_{12} & Z_{13} & \ldots & Z_{1 n} \\
Z_{21} & Z_{22} & Z_{23} & \ldots & Z_{2 n} \\
\mathrm{M} & \mathrm{M} & \mathrm{M} & \mathrm{M} & \mathrm{M} \\
Z_{n 1} & Z_{n 2} & Z_{n 3} & \ldots & Z_{n n}
\end{array}\right)
$$

Taking mutual coupling into account, the channel matrix $\mathrm{H}$ is modified to $\mathrm{H}_{\mathrm{MC}}$. The overall MIMO input-output relationship will be $[3,5]$ :

$$
y=H_{M C} x+n
$$

where $\mathrm{H}_{M C}=\mathrm{C}_{\mathrm{R}} \mathrm{HC}_{\mathrm{T}}$ is the modified channel matrix and $\mathrm{C}_{\mathrm{R}}$ and $\mathrm{C}_{\mathrm{T}}$ is the coupling matrix for transmitter and receiver respectively

In order to investigate the effect of inter-element distance and mutual coupling, channel matrix should be properly normalized. There are two main normalization methods [3]. The first one is called the Forbenius norm which normalizes $\mathrm{H}_{\mathrm{MC}}$ such that:

$$
\left\|H_{M C}\right\|_{F}^{2}=N_{T} N_{R}
$$

This normalization is performed on each realization of the channel matrix, which includes the propagation channel and antennas. It is limited because the differences in the channel gain due to antennas are not taken into account. On the other hand, this type of normalization permits investigation of correlation between the channel matrix entries and gives good indication of the richness of the multipath environment.

The second one is also called Forbenius norm which normalizes $\mathrm{H}$ as follows

$$
\|H\|_{F}^{2}=N_{T} N_{R}
$$

This normalization is also performed on each realization of the channel matrix, but it includes the propagation channel only. This normalization permits investigation to the effect of the instantaneous changes of received power due to mutual coupling.

\section{Channel Capacity:}

Two channel scenarios are considered. The first one is considered when the channel 
state information is known only at the receiver and the channel is i.i.d. Rayleigh fading, then uniform transmitting power is optimal at the transmitter $[3,5]$. In this case, the capacity is given by [3]

$$
C_{E P}=\log _{2}\left[\operatorname{det}\left(I+\frac{\rho}{N_{T}} H H^{\dagger}\right)\right] b p s / H z
$$

where $\mathrm{C}_{\mathrm{EP}}$ is the waterfilling capacity, det (.) denotes the determinant of a matrix, $\mathrm{I}$ is an identity matrix $\left(\mathrm{N}_{\mathrm{R}} \times \mathrm{N}_{\mathrm{T}}\right), \rho$ is the average received Signal to Noise Ratio (SNR), and $\mathrm{H}^{\dagger}$ is the complex conjugate transpose of $\mathrm{H}$.

If the channel state information known at both the transmitter and receiver and the channel is independent and identically distributed (i.i.d.) Rayleigh fading, then waterfilling is optimal at the transmitter [3,5]. The resulting capacity is given by [3]

$$
C_{W F}=\sum_{i=1}^{m} \log _{2}\left(\mu \lambda_{i}^{-1}\right)^{+} \quad \text { bps } / H z
$$

where $\mathrm{C}_{\mathrm{WF}}$ is the waterfilling capacity and $\mu$ is chosen from the waterfilling algorithm, which is

$$
\rho=\sum_{i=1}^{m}\left(\mu-\lambda_{i}^{-1}\right)^{+}
$$

where $(\cdot)^{+}$for those terms which are positive and $\lambda_{1}, \lambda_{2}, \cdots, \lambda_{\mathrm{m}}$ are the eigenvalues of $\mathrm{HH}^{\dagger}$ with $\mathrm{m}=\min \left(\mathrm{N}_{\mathrm{T}}, \mathrm{N}_{\mathrm{R}}\right)$.

\section{Results and Discussion:}

In this section, the correlation and mean capacity of $4 \times 4$ MIMO system were analyzed using the one-ring channel model shown in Fig. 1. Ansoft Designer software is used in analysis of the antenna array illustrated in Fig. 2. The proposed scenario considers the MS has $\mathrm{N}_{\mathrm{T}}=4$, and is placed at broadside relative to $\mathrm{N}_{\mathrm{R}}=4 \mathrm{BS}$-array elements. The distance between both stations (D) is $300 \lambda$. For each channel realization, a total of 100 scatterers are placed randomly and uniformly distributed on a disc of radius $\mathrm{R}=200 \lambda$, centered on the mobile. The mobile has a total power $\rho=20 \mathrm{~dB}$ and 100000 channel realizations were generated for each value of element spacing at MS $\left(\mathrm{d}_{\mathrm{m}}\right)$ to compute the 
required statistics.

The simulation was done using the equal power (EP) and waterfilling (WF) schemes for a fixed interelement spacing between BS antenna $\left(\mathrm{d}_{\mathrm{b}}\right)$ equal $0.5 \lambda$ while the MS antenna interelement spacing is varied over $\left(0.3 \lambda \leq \mathrm{d}_{\mathrm{m}} \leq 1 \lambda\right)$ for conventional substrate and $\left(0.47 \lambda \leq \mathrm{d}_{\mathrm{m}} \leq 1 \lambda\right)$ for spiral EBG substrate.

The effect of coupling on the correlation between paths $E\left[h_{1,1}, h_{1,2}\right]$ with and without spiral EBG structure is shown in Fig. 4 for $d_{m}$. The correlation between these two channel coefficients without mutual coupling (ideal case) is exactly the same as the results reported in [5]. This figure illustrates the effect of the coupling on the correlation with and without spiral EBG with element spacing $d_{m}$ in the range $(0.31 \lambda-0.6 \lambda)$. It is clear that it has no effect in the range $\left(0.6 \lambda<\mathrm{d}_{\mathrm{m}} \leq 1 \lambda\right)$. This result agrees with that indicated in Fig. 3 where the mutual coupling is nearly the same with and without spiral EBG for $d_{m} \geq 0.6 \lambda$. It can also be noted that in the range $\left(0.48 \lambda \leq d_{m} \leq 0.6 \lambda\right)$, which is suitable for the design of antenna arrays, the mutual coupling reduces the correlation level by about 0.15 . Spiral EBG reduces the MC leading to increase the correlation level and will be as the ideal case at $d \approx 0.48 \lambda$.

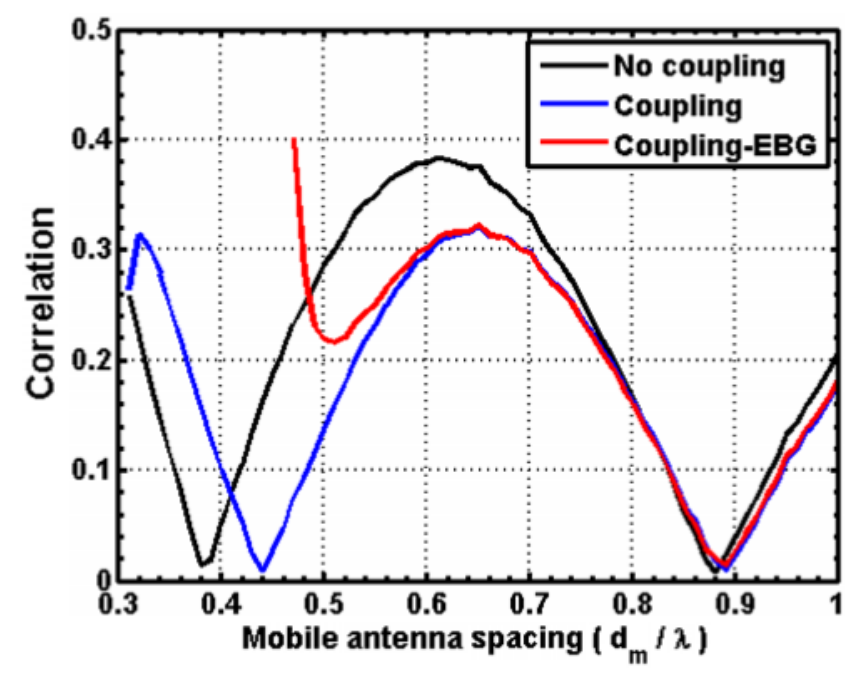

Figure (4): Mutual coupling effect on the correlation between paths $E\left[h_{1,1}, h_{1,2}\right]$ versus different element spacing $d_{m}$ at the mobile. The base station separation is $d_{b}=0.5 \lambda$ and the scatter disc radius $R=200 \lambda$

Figure 5 shows the effect of the mutual coupling on the mean capacity of the $4 \times 4$ MIMO system with and without spiral EBG structure. This figure reveals that both EP and WF 
results show the same trend. For both cases the mutual coupling decreases the capacity by $(0.1-0.42)$ bits $/ \mathrm{s} / \mathrm{Hz}$ as shown in Fig.6. The prior work [4] obtain $0.45 \mathrm{~b} / \mathrm{s} / \mathrm{Hz}$ drop in capacity due to coupling. Using spiral EBG structure improves the capacity such that its value is very close to the ideal case with a small difference of about $\pm 0.1 \mathrm{~b} / \mathrm{s} / \mathrm{Hz}$. At separation of $0.5 \lambda$ at the transmitting and receiving ends, the mean capacity considers spiral EBG structure reaches the ideal case capacity.
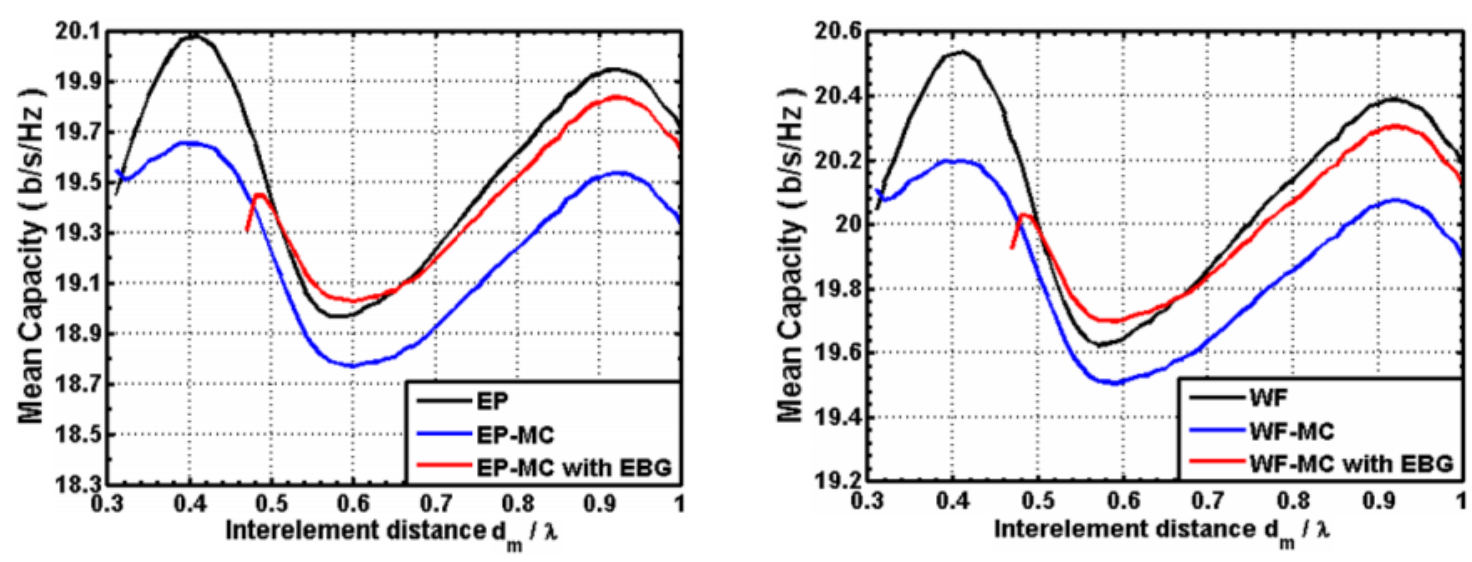

Figure (5): Mutual coupling effect on the mean capacity vs. interelement spacing $\left(d_{m}\right)$ with $d_{b}=0.5 \lambda$ and $S N R=20 \mathrm{~dB}$ for one-ring channel model using equal power and waterfilling schemes

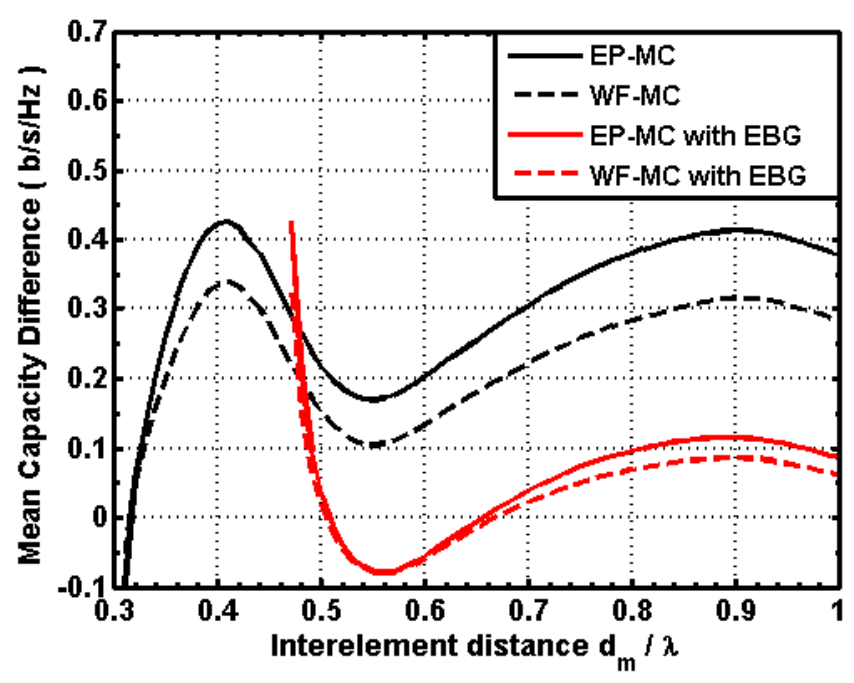

Figure (6): Difference between mean capacity of mutual coupling with and without spiral EBG structure and mean capacity of the ideal case for EP and WF schemes

\section{Conclusions:}


The effects of mutual coupling with and without spiral EBG structure on the correlation and mean capacity of $4 \times 4$ MIMO system have been studied. A linear array formed by microstrip patch antennas has been considered both for the transmitting and receiving ends. The simulation of the array was carried out by Ansoft Designer simulator to obtain the coupling matrices with and without the spiral EBG structure. These matrices are used in the analysis of the MIMO system using the one-ring channel model for EP and $\mathrm{WF}$ schemes at $\mathrm{SNR}=20 \mathrm{~dB}$. The results demonstrated that a maximum reduction of 17 $\mathrm{dB}$ in mutual coupling can be obtained using 2 columns and 4 rows of spiral EBG structure. The mutual coupling reduces the correlation level between paths by around 0.15 for the element separation range $(0.48 \lambda-0.6 \lambda)$, which is suitable for the design of antenna arrays. In this range, using spiral EBG increases the correlation level such that the difference from the ideal case reaches almost zero at $\mathrm{d}_{\mathrm{m}} \approx 0.48 \lambda$. Using spiral EBG structure improves the capacity such that its value is very close to the ideal case with a small difference of about $\pm 0.1 \mathrm{~b} / \mathrm{s} / \mathrm{Hz}$. At separation of $0.5 \lambda$ at the transmitting and receiving ends, the mean capacity with spiral EBG structure reaches the ideal case capacity.

\section{References:}

[1] C. Kalialakis, A. Collado, and A. Georgiadis, "Capacity of Linear Rectangular Microstrip Antenna Arrays,"Antennas and Propagation, EuCAP 2009, $3^{\text {rd }}$ European Conference, pp. 3114-3117, Berlin, Germany, 23-27 March, 2009.

[2] C. Poongodi and Dr. A. Shanmugam, "Capacity of MIMO Antenna Configurations for Spatial Channel and One Ring Model Scenarios," 2010 International Journal of Computer Applications (0975 - 8887), vol. 1, No. 12, pp. 81-85.

[3] S. Pan, S. Durrani, and M. E. Bialkowski, "MIMO Capacity for Spatial Channel Model Scenarios," Australian Communication Theory Workshop, pp. 25-29, Adelaide, Ausralia, 5-7 February, 2007.

[4] E.Michailidis, C. Tsimenidis and G. Chester, "Mutual Coupling Reduction in a Linear Two Element Patch Array and its Effect on Theoritical MIMO Capacity, " Antennas and Propagation Conference, LAPC 2008, pp. 457-460, Loughborough, England, 17-18 March, 2008. 
[5] T. Svantesson, and A. Ranheim, "Mutual Coupling Effects on the Capacity of Multielement Antenna Systems, " in Proc. IEEE Int. Conf. on Acoustics, Speech and Signal Processing, IEEE ICASSP '01, vol.4, pp. 2485-2488, Salt Lake, UT, USA, 7-11 May 2001.

[6] M. M. Aldemerdash, A. M. Abdin, A. A. Metxees and H. A. Elmikati, "Effect of Mutual Coupling on the Performance of Four elements Microstrip Antenna Array Fed by a Butler Matrix, " $29^{\text {th }}$ National Radio Science Conference, NRC 2012, pp. 127-140, Cairo university, Egypt, 10-12 April, 2012. 